\title{
Mutual coupling compensation of the conformal array antenna based on the active element pattern method
}

\author{
Zhenzhen Li, Yuwen Wang, Zhili Wang \\ Aircraft Technology School of Astonautics and Aeronautics, UESTC Chengdu, China \\ 742883363@qq.com, 83211668@qq.com, 1624814255@qq.com
}

Keywords: conformal antenna array; mutual coupling; active element pattern

\begin{abstract}
Due to the mutual coupling of conformal array cannot be ignored, this article proposes a simple compensation method for the mutual coupling compensation. The method calculates he far field direction diagram of the array by Simplifying array element classification, and then obtains the correction with the relationship between the isolation unit pattern and the active pattern, in the end, by obtaining correct incentives compensates the array. The simulation results show that this method is better compensated the error which the mutual coupling brings.
\end{abstract}

\section{Introduction}

The conformal array antenna array is to install the array element on the surface of the carrier, the carrier space. Because of the influence of the vector space, the antenna array element spacing is small. When the mutual coupling effect is significant, the Radiation characteristics of array antenna unit will change, Serious will cause the orientation image distortion.

The active unit direction graph method is quite popular in recent years, especially in the analysis and synthesis of the large array antenna and the conformal arrays analysis and synthesis of conformal arrays. The concept of the active element pattern (AEP) firstly was proposed by Hansen in 1966, which means the far field pattern about only motivating a reference unit and other units by matching load in array environment. Different from common impedance method, the equivalent network, the method of moments, such as simulated annealing algorithm [1-3], the active element pattern method under the certain accuracy is more simple and small amount of calculation, more practical especially in the large array.

This article uses the microstrip patch antenna conformal array as a model. It uses a full-wave electromagnetic field numerical analysis and the improved active element pattern method for mutual coupling compensation. It is classified according to the array element position in order to simplify the active unit pattern to solve the total far field pattern of array. It is extracted the mutual coupling coefficient matrix according to the relationship between the active element pattern and isolated element. The revised incentives can be obtained by the mutual coupling coefficient, and then the revised total far field pattern of the array can be obtained by the revised incentives.

\section{The conformal array modeling}

For airborne conformal array antenna, it uses sub-arrays modeling analysis for the convenience of analysis. As Fig.1, a plan for the $1 \times 12$ conformal microstrip patch antenna array arrangement diagram and side structure diagram, array elements use the coaxial feed, $\mathrm{N}=12$, normal operating frequency is $\mathrm{f}_{0}=2.45 \mathrm{GHz}$, the distance between array elements is $d=0.5 \lambda$, unit patch dielectric layer is $\varepsilon_{r}=4.4$.

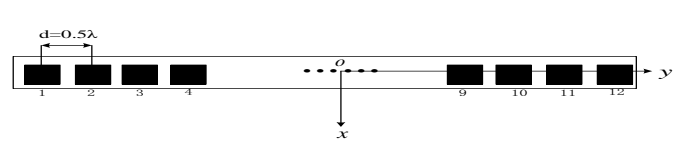

(a) Top view

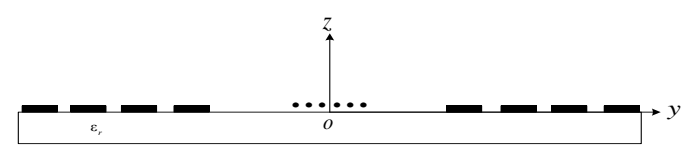

(b) Side view

Figure 1 Array model 


\section{Conformal array far field synthesis based on the active unit pattern}

Because HFSS electromagnetic simulation platform can quickly realize a small array simulation, but it is difficult to directly simulate a large antenna array because of consuming too much. Fig. 2 shows the basic agreement to the same position picket active unit pattern.
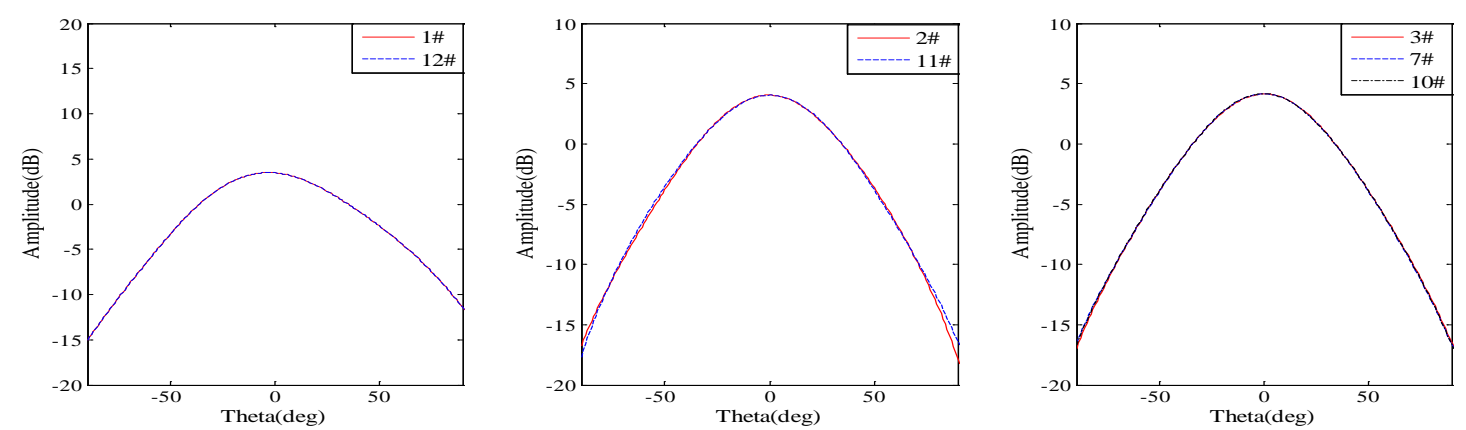

Figure 2 Active element patterns in different positions

According to array array element position, the array element is divided into the inner element, neighbor element, boundary element, as shown Fig.3.

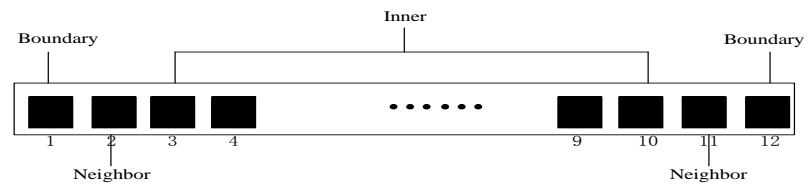

Figure 3 Classification of 12 elements array

According to the traditional pattern of the active principle, the general direction is determined by the numerical superposition of active pattern about each array element in the array antenna[4,5].Assume that active element pattern of each array element has been obtained, the total far-filed radiation expression of array is:

$$
\boldsymbol{E}(\theta, \varphi)=\sum_{m=0}^{N-1} I_{m} e^{\mathrm{j} \phi_{m}} \boldsymbol{F}_{m}^{e}(\theta, \varphi) \exp \{\mathrm{j} k m d \cos \varphi \sin \theta\}
$$

In the Eq.1, $I_{m}, \varphi_{m}$ respectively express the added incentive of amplitude and phase on the array element whose number is $\mathrm{m} . \boldsymbol{F}_{m}^{e}(\theta, \varphi)$ is the active element pattern of the unit $\mathrm{m}$.

According to the above array element classification, the Eq.1 can be also expressed as:

$$
\boldsymbol{E}(\theta, \varphi)=\boldsymbol{E}_{\mathrm{I}}(\theta, \varphi)+\boldsymbol{E}_{N}(\theta, \varphi)+\boldsymbol{E}_{B}(\theta, \varphi)
$$

$\boldsymbol{E}_{\mathrm{I}}(\theta, \varphi), \boldsymbol{E}_{N}(\theta, \varphi), \boldsymbol{E}_{B}(\theta, \varphi)$ respectively composited by the active element pattern of the intermediate, adjacency, boundary elements.

\section{Mutual coupling coefficient of extraction and compensation}

When calculating the mutual coupling coefficient between the elements in the rules array, it usually can be used Fourier coefficient method according to its periodic. But this kind of method is not suitable for conformal array [6].

For array model shown in Fig.1, it is respectively obtained active array element pattern and array element pattern under isolation (acronym isolated pattern).

Assume that array element generates active radiation after motivating, the coupling effect which leads to becomes active coupling. Under the condition of active coupling, the matrix $\boldsymbol{F}^{e}, \boldsymbol{F}^{0}$ are respectively represented as active pattern matrix and isolated pattern matrix, $\boldsymbol{C}$ is represented as $\mathrm{N}$ order of coupling coefficient matrix. The relationship between active pattern and isolated direction pattern can be expressed as:

$$
\begin{gathered}
\boldsymbol{F}^{e}=\boldsymbol{C F}^{0} \\
\boldsymbol{F}^{e}=\left[F_{1}^{e}(\theta, \varphi), F_{2}^{e}(\theta, \varphi), \cdots, F_{N}^{e}(\theta, \varphi)\right]^{T}
\end{gathered}
$$




$$
\begin{aligned}
& \boldsymbol{F}^{0}=\left[F_{1}^{0}(\theta, \varphi), F_{2}^{0}(\theta, \varphi), \cdots, F_{N}^{0}(\theta, \varphi)\right]^{T} \\
& \boldsymbol{C}=\left[\begin{array}{cccc}
c_{11} & c_{12} & \cdots & c_{1 N} \\
c_{21} & c_{22} & \cdots & c_{1 N} \\
\vdots & \ddots & & \vdots \\
c_{N 1} & c_{N 2} & \cdots & c_{N N}
\end{array}\right]
\end{aligned}
$$

The above formula, $c_{i i}$ is represented as the $i$ unit of the coupling influence coefficient, $c_{i j}$ is represented as the mutual coupling effect coefficient between the $i$ unit and $j$ unit.

According to the Eq.3 the mutual coupling coefficient matrix can be calculated based on the least squares solution, namely:

$$
\boldsymbol{C}=\left(\left(\boldsymbol{F}^{0}\right)^{H} \boldsymbol{F}^{0}\right)^{-1}\left(\boldsymbol{F}^{0}\right)^{H} \boldsymbol{F}^{e}
$$

Without the mutual coupling effect, the incentive coefficient matrix is expressed as:

$$
W=\left[w_{1} w_{2} \cdots w_{N}\right]
$$

The amendment of the mutual coupling of $\mathrm{N}$ elements conformal array pattern can be expressed as:

$$
\boldsymbol{F}=\boldsymbol{W F}^{0}=\left(\boldsymbol{W C}^{-1}\right) \boldsymbol{F}^{e}
$$

In Eq.9 , $\left(w_{C^{-1}}\right)$ represents the correction of the mutual coupling.

\section{The calculation and simulation results}

1) Validation of AEP method after array element classification

For antenna array model as shown in Fig.1, through changing the scanning angle, the total active pattern based on classification of elements and full-wave simulation pattern by HFSS are obtained respectively. As can be seen from Fig.4, when the scan angle $\varphi=0^{\circ}$, the difference of gain is only $0.03 \mathrm{~dB}$, maximum difference of side-lobe level is only $0.02 \mathrm{~dB}$. Both are very consistent.

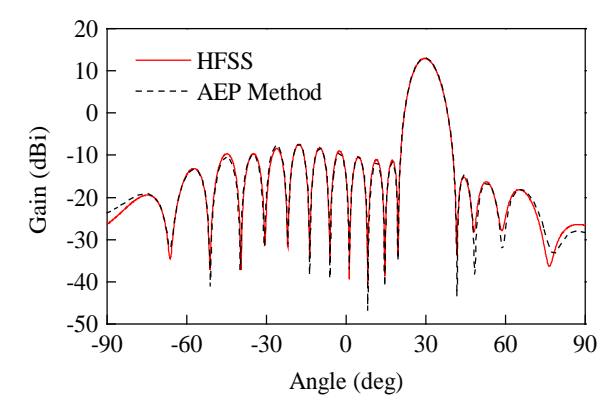

(a) $\varphi=30^{\circ}$

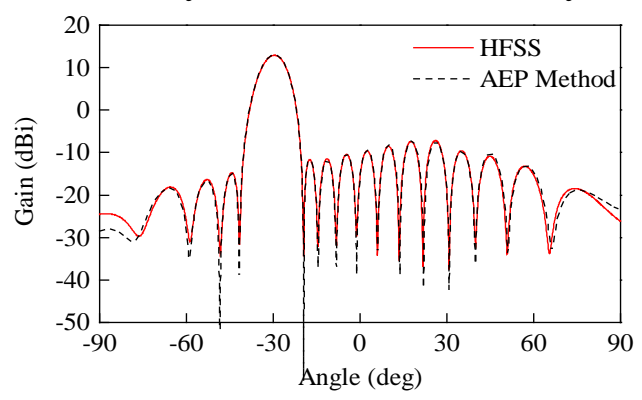

(b) $\varphi=-30^{\circ}$

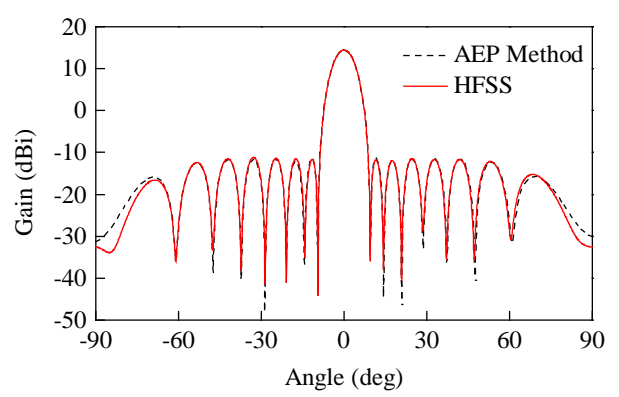

(c) $\varphi=0^{\circ}$

Figure 4 Gain pattern as the scanning angle change

2) Mutual coupling coefficient

On a flat space of sampling points to 361, mutual coupling coefficient matrix can be get achieved, wherein the variation of mutual coupling coefficients $\mathrm{c}_{m 3}$ and $\mathrm{c}_{m 7}$ shown in Fig.5. From the figure can get conclusion, the greatest impact is self-coupling effect of the element $\mathrm{M}$, followed 
by mutual coupling between $\mathrm{M}$ and its two adjacent elements.

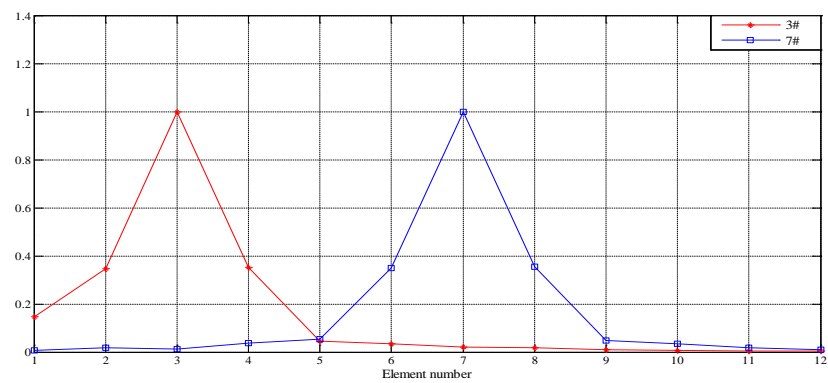

Figure 5 Mutual coupling coefficients of element 3\# and 7\#

3) Corrected excitation

Amplitude and phase characteristics of corrected excitation as shown in Fig.6. Analysis of the picture can be draw the conclusion that the influence of mutual coupling among inner elements is biggest, and away from the center of the array by boundary element mutual coupling minimized. At the same time, the maximum difference of corrected phase than the previous is $3^{\circ}$. That illustrates the impact of mutual coupling on the amplitude is bigger than the influence of the phase.

4) Mutual coupling compensation of array pattern

Array pattern after compensation is shown in Fig.7. The side lobe level is $-13.6 \mathrm{~dB}$ under the consideration of the mutual coupling, compared with the mutual coupling arrays (namely, isolated element pattern) under higher sidelobe level $2 \mathrm{~dB}$. The sidelobe level after compensated dropped to $15.58 \mathrm{~dB}$, compared with non-state of mutual coupling difference $0.02 \mathrm{~dB}$. So compensation effect is more ideal.
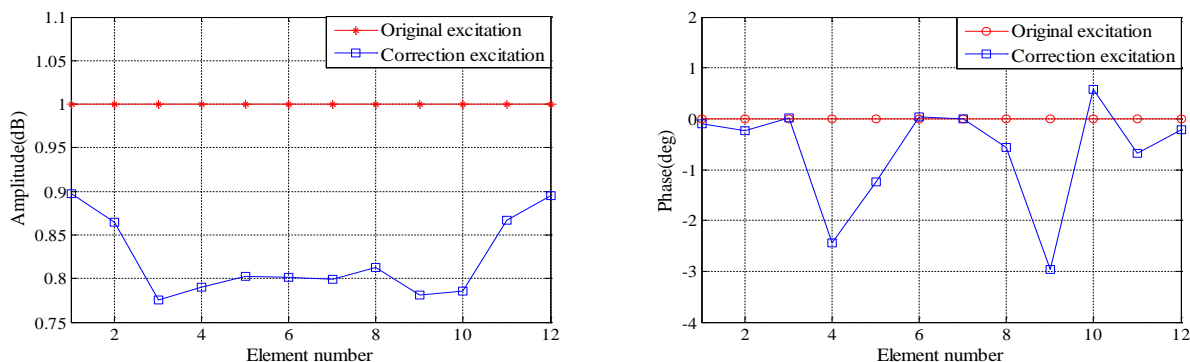

Figure 6 Characteristics of corrected excitation amplitude and phase

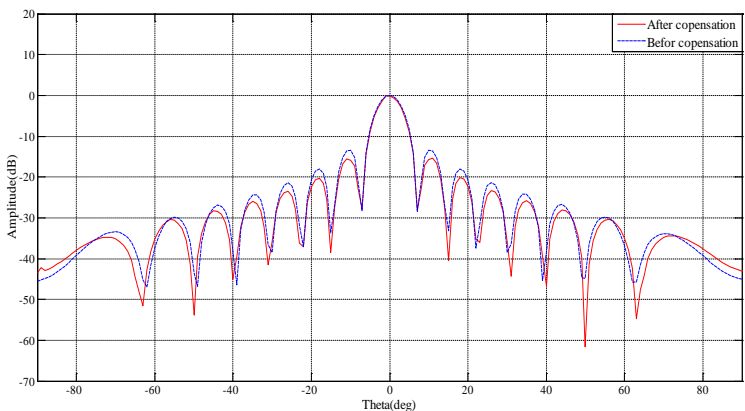

Figure 7 Mutual coupling array pattern before and after correction

\section{Conclusion}

A simple method is proposed in this paper in view of the conformal array antenna mutual coupling compensation. In the method, we classify the elements based on the traditional active element pattern, then achieve the total active pattern. Then mutual coupling coefficients and corrected excitation can be obtained rapidly according to the relationship between the active pattern and isolated pattern, and combined with the least squares method. The array mutual coupling correction has been completed by the corrected excitation and active pattern. This method is suitable for the large array antenna, simplifies the compensation process, and avoids a lot of iterative calculation. And it can achieve high compensation precision. 


\section{References}

[1] Pozar, D.M.: The Active Element Pattern. IEEE Transactions on Antennas and Propagation, Aug. 1994, Vol.42(8), pp.1176-1178

[2] Yao Wang: Compensation of Mutual Coupling and Array Antenna Technology, IEEE Transactions on Antennas and Propagation, Apr. 2008

[3] Guanjun Lan, Junping Shang, Yingbo Deng: Analysis of Antenna Array Moment Method .Electronic Technology, 2008,21 (8): 14-16

[4] Qing-Qiang He, Yu-Hong Liang. Pattern Synthesis and Polarization Optimization of a Conical Array[J]. Journal of Electronic Science and Technology,2011,01:71-77

[5] Yang Xs , Qian H. Radiation Pattern Computation of Pyramidal Conformal Antenna Array with Active-Element Pattern Technique. IEEE Antennas And Propagation Magazine, 2011 Feb, Vol.53(1), pp.28-37

[6] Fei Zhao, Huiying Qi, Ke Xiao, etal. The Experimental Study of Mutual Coupling in Conformal Array [J]. Microwave and Optical Technology Letters. 2012, 54(1): 199-203. 